\title{
Effect of bradykinin on renal mesangial cell proliferation and extracellular matrix secretion
}

\author{
C.Y. Liu, L.L. Zhou, Q. Cheng, S.N. Jiang, J. Sheng, J.D. Sun and J.Y. Zhao \\ Department of Nephrology, Second Hospital of Dalian Medical University, \\ Shahekou District, Dalian Liaoning Province, China \\ Corresponding author: J.Y. Zhao \\ E-mail: jiuyangzhao@126.com \\ Genet. Mol. Res. 13 (1): 490-498 (2014) \\ Received August 26, 2013 \\ Accepted October 26, 2013 \\ Published January 21, 2014 \\ DOI http://dx.doi.org/10.4238/2014.January.21.18
}

\begin{abstract}
Recent studies have found that bradykinin (BK) plays a role in delaying glomerulosclerosis, although the mechanism of this phenomenon remains unclear. Mesangial cell proliferation (MCP) and extracellular matrix (ECM) secretion are important mechanisms for glomerulosclerosis. This study investigated the impact of BK on the platelet-derived growth factor (PDGF)-induced proliferation of mesangial cells, and evaluated its correlations with the extracellular signal-related kinase (ERK) signaling pathway. The results showed that on its own, 10-1000 $\mu \mathrm{g} / \mathrm{L}$ BK promoted MCP and ECM secretion and induced ERK phosphorylation. However, BK administration after PDGF pre-incubation inhibited PDGF-induced MCP, ECM secretion, and ERK phosphorylation. The BK B2 receptor-specific antagonist, HOE-140, and tyrosine phosphatase inhibitor (OV) effectively blocked the function of $\mathrm{BK}$. In summary, these results demonstrated that BK has a bidirectional effect on MCP and ECM secretion: when used alone, it promoted effects on these phenomena, but these effects were inhibited when combined with PDGF. This suggests that the role of BK might be achieved through inhibiting activation of the PDGF-induced ERK1/2 pathway.
\end{abstract}

Key words: Bradykinin; Platelet-derived growth factor; Mesangial cells; Extracellular signal-regulated kinase 


\section{INTRODUCTION}

Mesangial cell proliferation (MCP) and extracellular matrix (ECM) secretion are key steps in glomerulosclerosis. Identifying methods of preventing the proliferation of mesangial cells and blocking their secretions has undeniable clinical significance for preventing the occurrence and development of kidney disease, and for delaying the deterioration of renal function.

The kallikrein-kinin system (KKS) is a complex multi-enzyme system that is widespread across various tissues and shows a variety of biological activities (Wright et al., 2008; Costa-Neto et al., 2008; Tang et al., 2011; Phipps et al., 2012). Recent studies have found that the KKS could significantly inhibit glomerulosclerosis (Schanstra et al., 2002; Marceau and Regoli, 2004; Kakoki et al., 2004; Tan et al., 2005; Tomita et al., 2012). The kidney contains various components of KKS, and bradykinin (BK), the most important bioactive peptide of KKS, is the final effector exerting the effects described above. The presence of BK receptors at the surface of mesangial cells has been confirmed, and the B2 receptor of BK shows particularly high expression (Bascands et al., 1993). Glomerulosclerosis is a common developmental process of chronic kidney disease, which mainly acts to increase MCP and ECM secretion. Therefore, investigating the effects of BK on MCP might increase understanding of the pathogenesis and treatment of glomerulosclerosis. Currently, the effects of BK on mesangial cells remain controversial (Tang et al., 2011). The roles that BK plays in its physiological state have been suggested to differ from those in pathological states. During glomerulosclerosis, increased expression of growth factors plays an important role in MCP. Therefore, we hypothesized that the roles of BK in mesangial cells, on MCP in particular, might be different when interacting with growth factors. The platelet-derived growth factor (PDGF) is an important cytokine. Using PDGF to stimulate mesangial cells and to stimulate MCP in vivo, we studied the effects of BK on MCP in the pathological state. Because the extracellular signal-related kinase (ERK) is an important PDGF downstream signaling pathway, we speculated that BK might mediate PDGF-stimulated MCP via the ERK signaling pathway. In this study, mesangial cells cultured in vitro were used to evaluate the difference between the role of BK alone and in combination with PDGF, with the aim of providing new evidence for BK in the occurrence and development of kidney disease.

\section{MATERIAL AND METHODS}

\section{Reagents}

BK was obtained from the Calbiochem company (USA), PDGF-BB was obtained from PeproTech Inc. (New Jersey, USA), the BK B2 receptor-specific antagonist, HOE-140, and the tyrosine phosphatase inhibitor, OV, were purchased from Sigma (USA), Type I and IV collagen (Col I and Col IV) enzyme-linked immunosorbent assay (ELISA) kits were obtained from the Shanghai Senxiong Company, and ERK, phosphorylated ERK (p-ERK), and glyceraldehyde-3-phosphate dehydrogenase (GAPDH) antibodies were purchased from Cell Signaling Technology.

\section{Cell culture and general experimental methods}

Immortalized rat mesangial cells were purchased from CAMBREX (USA), and were 
cultured in $10 \%$ fetal calf serum (FCS)-supplemented RPMI 1640 culture medium at $37^{\circ} \mathrm{C}$ in a $5 \% \mathrm{CO}_{2}$ incubator. When reaching $60-70 \%$ confluence, mesangial cells were further cultured for $24 \mathrm{~h}$ in FCS-free RPMI 1640 culture medium before initiating the experiment to achieve cell synchronization. Cells were then incubated with $0,10,100$, and $1000 \mu \mathrm{g} / \mathrm{L}$ BK for $48 \mathrm{~h}$. The methyl tetrazolium (MTT) assay was applied to detect cell proliferation, and the supernatant was collected to detect the concentrations of $\mathrm{Col} \mathrm{I}$ and $\mathrm{Col}$ IV by ELISA. After mesangial cells were pre-incubated with $20 \mu \mathrm{g} / \mathrm{L}$ PDGF-BB for $1 \mathrm{~h}$, they were co-incubated with 0,10 , 100 , and $1000 \mu \mathrm{g} / \mathrm{L}$ BK for $48 \mathrm{~h}$. Cell proliferation was detected using the MTT assay, and the supernatant was collected to detect the concentrations of Col I and Col IV by ELISA. Different concentrations of BK $(0,10,100$, and $1000 \mu \mathrm{g} / \mathrm{L})$ were applied to cells for $2 \mathrm{~min}$, either alone or after $20 \mu \mathrm{g} / \mathrm{L}$ PDGF-BB pre-incubation for $1 \mathrm{~h}$. Cells were collected, total proteins were extracted, and the Western blot assay was used to detect the level of p-ERK. After preincubating with $1 \mu \mathrm{M}$ HOE-140 and $0.5 \mathrm{mM}$ OV for $1 \mathrm{~h}, 20 \mu \mathrm{g} / \mathrm{L}$ PDGF-BB was added to the pre-incubation solution with the cells for $1 \mathrm{~h}$ further, and then $100 \mu \mathrm{g} / \mathrm{L} \mathrm{BK}$ was added to co-incubate for $2 \mathrm{~min}$. Cells were then collected and total proteins were extracted to detect the p-ERK levels by Western blot analysis.

\section{Cell proliferation detection by MTT assay}

Cells were added onto 96-well plates at a density of $1 \times 10^{4}$ cells $/$ well $\left(10^{5}\right.$ cells $\left./ \mathrm{mL}\right)$, and were cultured without serum for $24 \mathrm{~h}$. After being subjected to different treatments, these cells were cultured for $48 \mathrm{~h}$, and were then co-incubated with $10 \mu \mathrm{L} 500 \mathrm{mg} / \mathrm{L}$ MTT in an incubator for $4 \mathrm{~h}$. The supernatant was removed and $100 \mu \mathrm{L}$ dimethyl sulfoxide was added. After shocking, the absorbance value was detected at $490 \mathrm{~nm}$ using a microplate reader. Each group had four parallel wells, and the detection was repeated three times to obtain the mean value of each group, which was used as the result for calculations and statistical analysis.

\section{ELISA test for Col IV content in the supernatant}

Cells were seeded on 96-well plates at a density of $1 \times 10^{4}$ cells/well. After being cultured in serum-free medium for $24 \mathrm{~h}$, different concentrations of BK $\left(10-10^{3} \mathrm{ng} / \mathrm{mL}\right)$ were added, cells were pre-incubated for $1 \mathrm{~h}$, and then PDGF-BB was added and cells were incubated for a further $48 \mathrm{~h}$. The supernatant was removed and used to determine the Col IV concentration by ELISA.

\section{Determination of total and p-ERK levels}

The Western blotting method was used to determine protein contents. Mesangial cells were seeded on $10-\mathrm{cm}$ Petri dishes, grown to $60-70 \%$ confluence, and were then cultured for $24 \mathrm{~h}$ in serum-free medium for synchronization. Total proteins were extracted under different treatments. The medium was removed and washed with ice-cold phosphate-buffered saline containing $1 \mathrm{mM} \mathrm{NaVO}_{4}$ three times to terminate the reaction. The protein lysis buffer (10 $\mathrm{mM}$ Tris-HCl, pH 7.4, $150 \mathrm{mM} \mathrm{NaCl}, 1 \mathrm{mM}$ EDTA, $0.2 \mathrm{mM} \mathrm{NaVO}_{4}, 1 \% \mathrm{NP}-40,1 \%$ Triton$\mathrm{X} 100,40 \mu \mathrm{g} / \mathrm{mL}$ PMSF, $10 \mu \mathrm{g} / \mathrm{mL}$ bright aprotinin, and $10 \mu \mathrm{g} / \mathrm{mL}$ aprotinin) was added (precooling), cells were scraped and transferred to the Eppendorf tube, and were then sonicated 
and centrifuged at $13,000 \mathrm{rpm}$ at $4^{\circ} \mathrm{C}$ for 20 min to obtain the supernatant. All of the above operations were performed on ice to avoid protein degradation. The protein concentration was determined based on the Bradford analysis method. Forty micrograms target protein sample was electrophoresed at a $270-\mathrm{mA}$ constant current at $4^{\circ} \mathrm{C}$, and then transferred to a polyvinylidene fluoride membrane. The reaction was terminated with the addition of $50 \mathrm{~g} / \mathrm{L}$ milk at room temperature for $2 \mathrm{~h}$, different primary antibodies were added, incubated overnight, the horseradish peroxidase-labeled secondary antibody (1:2000) was added, incubated for $2 \mathrm{~h}$ at room temperature, electrochemiluminescence solution was added and incubated for $1 \mathrm{~min}$, and then the films were developed in the darkroom. The absorbance value was determined and protein contents were calculated using GAPDH as an internal reference.

\section{Statistical analysis}

All data are reported as means $\pm \mathrm{SD}$, and the Student $t$-test was used for statistical analysis. Significant differences were indicated at $\mathrm{P}<0.05$.

\section{RESULTS}

\section{BK played a bidirectional role in $\mathrm{MCP}$}

A single application of $10-1000 \mu \mathrm{g} / \mathrm{L} \mathrm{BK}$ on its own significantly promoted the proliferation of mesangial cells in a dose-dependent manner. Concentrations of $20 \mu \mathrm{g} / \mathrm{L} \mathrm{BK}$ resulted in significant cell proliferation, and $1000 \mu \mathrm{g} / \mathrm{L} \mathrm{BK}$ induced double MCP compared to controls, as shown in Figure 1.

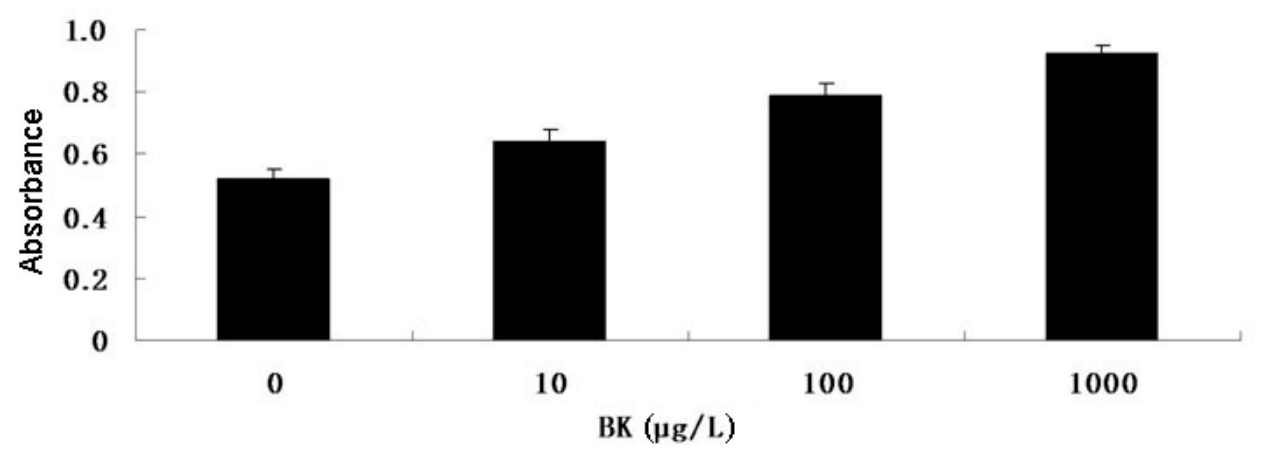

Figure 1. Proliferation of mesangial cells in different concentrations of bradykinin (BK) for $48 \mathrm{~h}$.

PDGF-BB resulted in significant MCP, as shown in Figure 2; however, various concentrations of $\mathrm{BK}$ significantly inhibited this proliferation $(\mathrm{P}<0.05)$ in a concentration-dependent manner compared with the PDGF alone group (Figure 2).

\section{BK played a bidirectional role in mesangial cell collagen secretion}

A single application of BK promoted Col I and Col IV secretion of mesangial cells in 
a dose-dependent manner, as shown in Figure 3. The highest BK concentration, $1000 \mu \mathrm{g} / \mathrm{L}$, resulted in a 10-fold increase in the amount of Col I and Col IV secreted by mesangial cells.

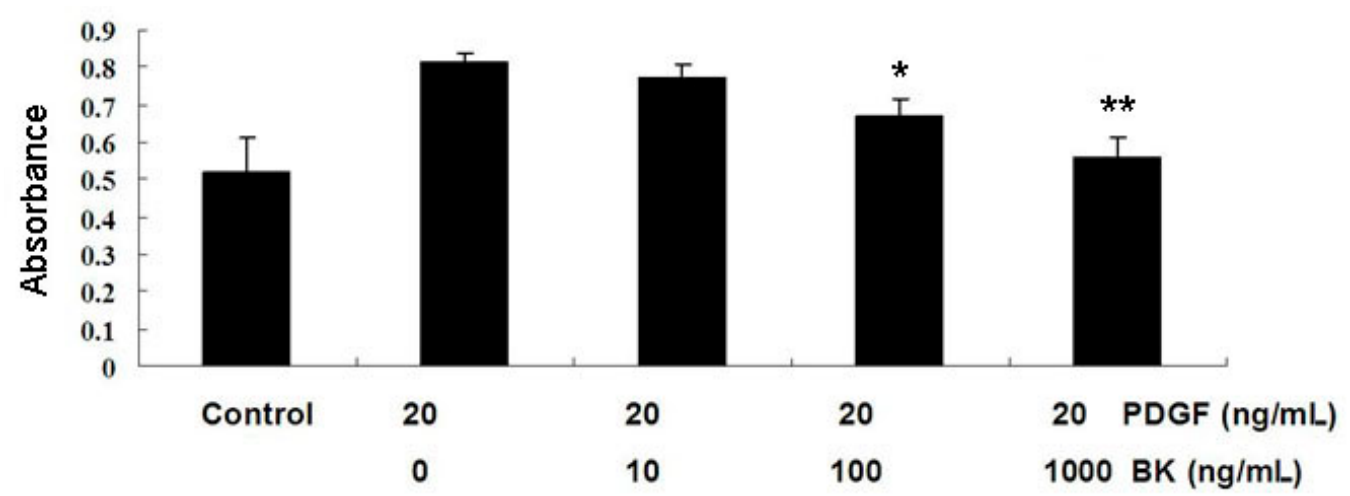

Figure 2. Effects of different concentrations of bradykinin (BK) and platelet-derived growth factor (PDGF) 20 $\mu \mathrm{g} / \mathrm{L}$ together on MCP for $48 \mathrm{~h} .{ }^{*} \mathrm{P}<0.05 ; * \mathrm{P}<0.01$.

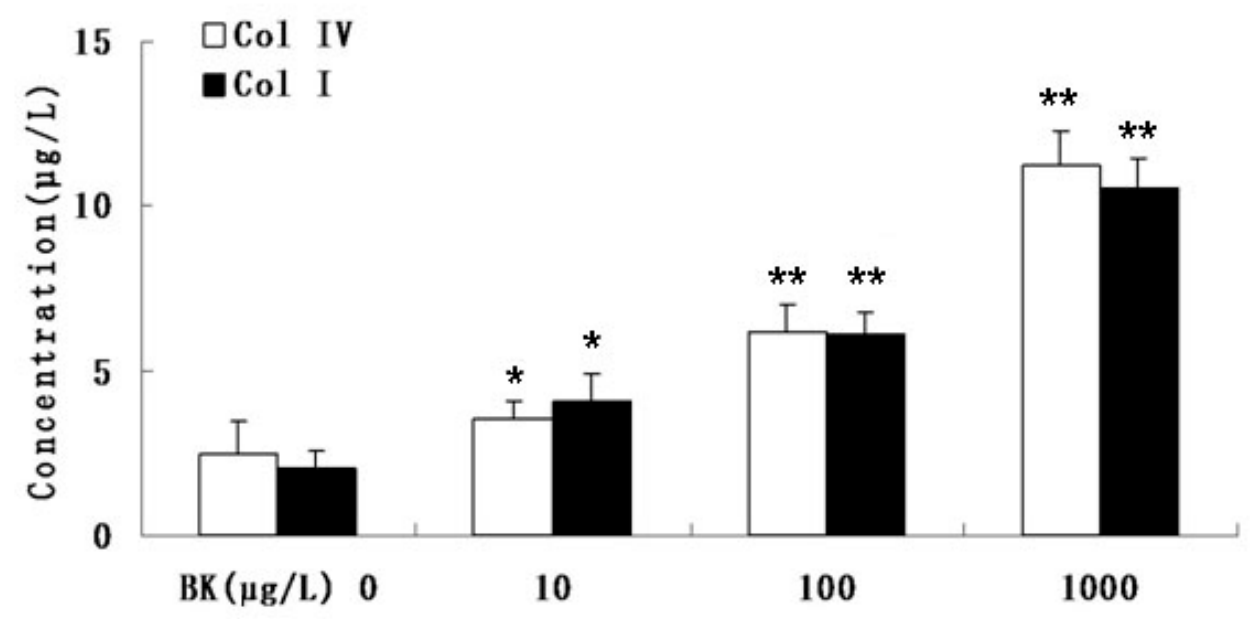

Figure 3. Mesangial cell collagen (Col I, Col IV) secretion in different concentrations of bradykinin (BK) at $48 \mathrm{~h}$. $* \mathrm{P}<0.05 ; * * \mathrm{P}<0.01$.

PDGF-BB also caused an increase in the secretion of Col I and Col IV from mesangial cells. PDGF-BB-induced Col IV secretion was only significantly inhibited (compared to the PDGF alone group) when cells were pre-incubated with BK before PDGF was added ( $\mathrm{P}<$ 0.05). As above, the effects of BK increased with increasing concentration (Figure 4).

\section{BK promoted mesangial cell ERK phosphorylation expression}

BK activated the ERK phosphorylation levels of mesangial cells. ERK phosphorylation levels gradually increased with increasing BK concentration (Figure 5). 


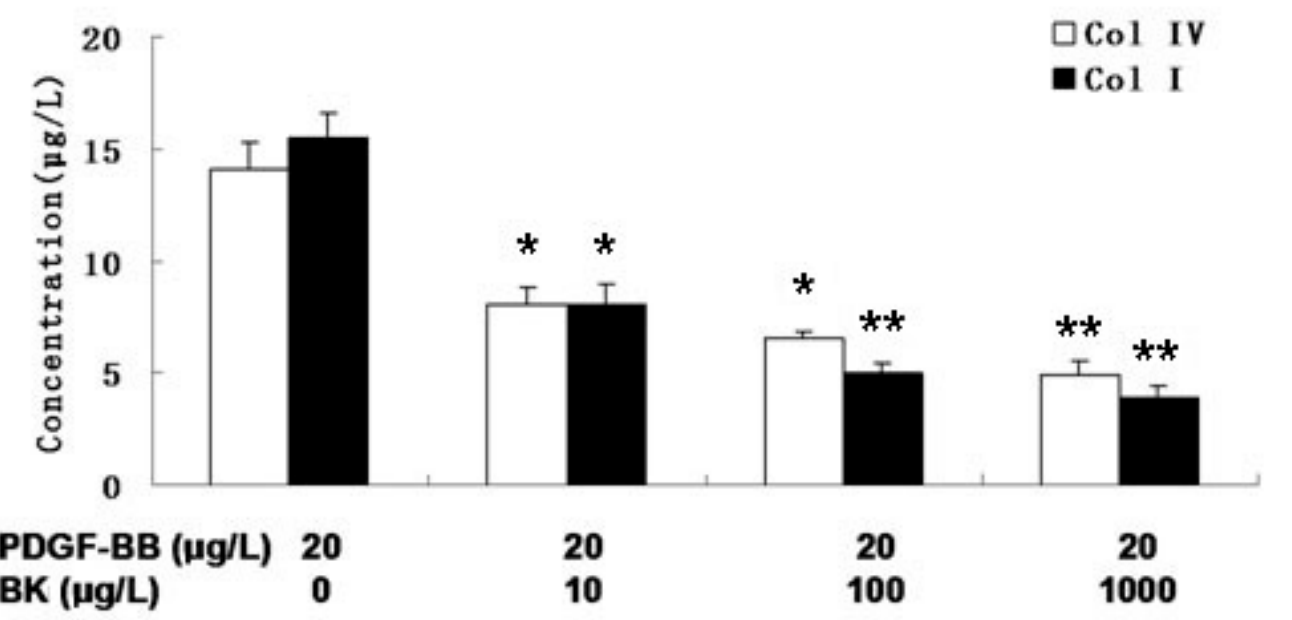

Figure 4. Effects of different concentrations of bradykinin (BK) and platelet-derived growth factor (PDGF) 20 $\mu \mathrm{g} / \mathrm{L}$ together on mesangial cell collagen (Col I, Col IV) secretion for $48 \mathrm{~h}$. $* \mathrm{P}<0.05 ; * * \mathrm{P}<0.01$.
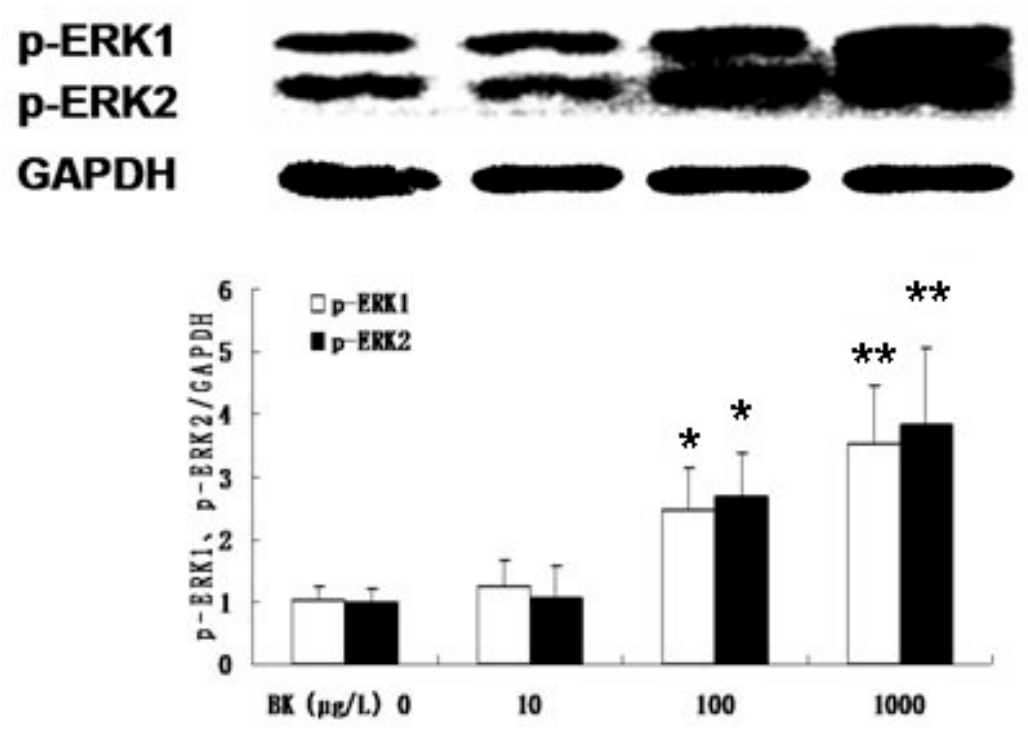

Figure 5. Effects of different concentrations of bradykinin (BK) on the levels of mesangial cell ERK phosphorylation (p-ERK). $* \mathrm{P}<0.05 ; * * \mathrm{P}<0.01$.

\section{BK inhibited PDGF-BB-induced mesangial cell ERK1/2 phosphorylation expression}

PDGF-BB also resulted in a significant increase of mesangial cell ERK1/2 phosphorylation levels compared to the control group $(\mathrm{P}<0.05)$; however, the effect was inhibited if cells were pre-incubated with BK before PDGF was added, as shown in Figure 6. 

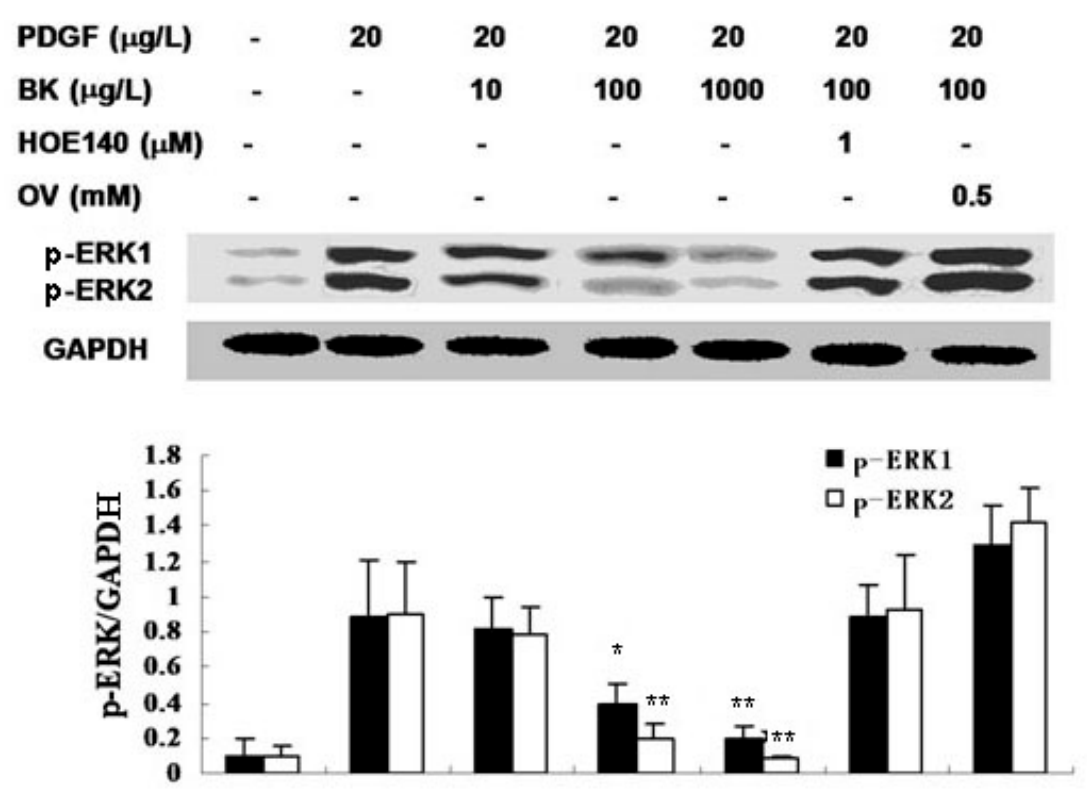

Figure 6. Effects of different concentrations of bradykinin (BK), platelet-derived growth factor (PDGF), HOE-140 and $\mathrm{OV}$ together on the levels of mesangial cell ERK phosphorylation (p-ERK). ${ }^{*} \mathrm{P}<0.05 ; * * \mathrm{P}<0.01$.

\section{Inhibition of PDGF-BB-induced mesangial cell expression of ERK1/2 phosphorylation by BK occurred via the BK receptor}

To clarify the underlying mechanism of the activity of BK, the receptor antagonist of BK, HOE-140, was prepared, and then BK and PDGF-BB were added. Inhibition of PDGFBB-activated expression of ERK1/2 phosphorylation significantly decreased after the addition of BK antagonists, which showed that the BK receptor was responsible for exerting the observed effects of BK, which was blocked with the addition of $1 \mu \mathrm{M}$ specific BK B2 receptor blocker. In addition, the tyrosine kinase inhibitor, OV, also blocked the effect of BK on PDGFinduced ERK phosphorylation (Figure 6).

\section{DISCUSSION}

Recent developments in molecular and cell biology have prompted increased interest in the role of the KKS. The KKS is one of the main pressure-releasing systems in the human body, and plays an important role in the regulation of vascular wall tension, cell proliferation, and matrix hyperplasia. It is composed of kininogen, kallikrein (KLK), and BK, of which the latter is the final effector molecule exerting the roles listed above. Previous studies using a replication-defective adenoviral vector to load the human KLK gene (Ad.CMV-CHK) have revealed that the KKS could inhibit damage such as glomerulosclerosis and proteinuria, renal toxicity tubule cell destruction (Murakami et al., 1998), and renal fibrosis (Zhang et al., 2004). These effects were independent of the effect of blood pressure lowering caused by the system, particularly in the case of diabetic nephropathy (Kakoki et al., 2004; Tan et 
al., 2007; Pawluczyk et al., 2006, 2008). BK is the effector peptide of KKS, and in vitro experiments have also confirmed that BK could inhibit MCP induced by FCS, growth factors, and high glucose (Alric et al., 2000; Blaes et al., 2012), as well as arterial smooth muscle cell proliferation that is induced by PDGF-AB. Several in-depth studies have recently emerged to further elucidate the mechanism of the above effects produced by the KKS, especially BK.

In the present study, BK, the ultimate effector peptide in the KKS, was applied directly to cells to observe its impact on MCP and ECM secretion in both resting and stimulated states. Results showed that BK was indeed two-direction adjustable, playing a promoting role in the resting state, and activating the mechanisms involved in the expression of phosphorylation of the ERK1/2 protein of the mitogen-activated protein kinase pathway, indicating that BK might play an important role in normal renal development. However, BK also played an inhibitory role in proliferation when cells were pre-stimulated with PDGF, as well as on PDGF-induced ERK phosphorylation. Blaes et al. (2012) also found that BK could inhibit the synthesis of mesangial cell Col I and Col IV that was induced by high-sugar, epithelial growth factor, and transforming growth factor- $\beta$. BK receptors are divided into two main categories, B1 and B2, with its main functions achieved through the B2 receptor (Kakoki et al., 2004; Tan et al., 2004; Abadir et al., 2006; Duka et al., 2006). In the present study, the blocker of the BK B2 receptor, HOE-140, inhibited the observed effects, indicating that the BK B2 receptor is involved in the negative effects of this cytokine. Additionally, the blockage of the tyrosine phosphorylation kinase inhibitor, OV, on the negative effect of BK resulted in increased activity of protein-tyrosine kinase in BK's anti-proliferative effect, suggesting that important loci located at the level of tyrosine phosphatase might be associated with the negative effects of BK. The BK B2 receptor is known to be located on the surface of membrane cells, and is a G-protein coupled receptor (GPCR). Since tyrosine kinase receptors (TPKCR) on the cell membrane result from the PDGF pathway, it appears likely that BK affected the TPKCR level when interacting negatively with other cytokines. The effects of BK might be related to novel protein-protein interaction mechanisms between GPCR and TPKCR, but this hypothesis awaits further confirmation.

This study demonstrated the bidirectional regulation of BK on MCP, explored the possible mechanisms for this effect by detecting ERK phosphorylation levels, and provided a theoretical basis for further research into the role of BK in mesangial cells.

\section{REFERENCES}

Abadir PM, Periasamy A, Carey RM and Siragy HM (2006). Angiotensin II type 2 receptor-bradykinin B2 receptor functional heterodimerization. Hypertension 48: 316-322.

Alric C, Pecher C, Schanstra JP, Bascands JL, et al. (2000). Bradykinin-induced inhibition of cell proliferation and tyrosine kinase activity in rat mesangial cells. Int. J. Mol. Med. 5: 85-93.

Bascands JL, Pecher C, Rouaud S, Emond C, et al. (1993). Evidence for existence of two distinct bradykinin receptors on rat mesangial cells. Am. J. Physiol. 264: F548-F556.

Blaes N, Pecher C, Mehrenberger M, Cellier E, et al. (2012). Bradykinin inhibits high glucose- and growth factor-induced collagen synthesis in mesangial cells through the B2-kinin receptor. Am. J. Physiol. Renal Physiol. 303: F293-F303.

Costa-Neto CM, Dillenburg-Pilla P, Heinrich TA, Parreiras-E-Silva LT, et al. (2008). Participation of kallikrein-kinin system in different pathologies. Int. Immunopharmacol. 8: 135-142.

Duka A, Duka I, Gao G, Shenouda S, et al. (2006). Role of bradykinin B1 and B2 receptors in normal blood pressure regulation. Am. J. Physiol. Endocrinol. Metab. 291: E268-E274.

Kakoki M, Takahashi N, Jennette JC and Smithies O (2004). Diabetic nephropathy is markedly enhanced in mice lacking the bradykinin B2 receptor. Proc. Natl. Acad. Sci. U. S. A. 101: 13302-13305. 
Marceau F and Regoli D (2004). Bradykinin receptor ligands: therapeutic perspectives. Nat. Rev. Drug Discov. 3: 845-852. Murakami H, Yayama K, Chao L and Chao J (1998). Human kallikrein gene delivery protects against gentamycin-induced nephrotoxicity in rats. Kidney Int. 53: 1305-1313.

Pawluczyk IZ, Patel SR and Harris KP (2006). Pharmacological enhancement of the kallikrein-kinin system promotes anti-fibrotic responses in human mesangial cells. Cell Physiol. Biochem. 18: 327-336.

Pawluczyk IZ, Tan EK, Lodwick D and Harris K (2008). Kallikrein gene 'knock-down' by small interfering RNA transfection induces a profibrotic phenotype in rat mesangial cells. J. Hypertens. 26: 93-101.

Phipps JA, Jobling AI, Greferath U, Fletcher EL, et al. (2012). Alternative pathways in the development of diabetic retinopathy: the renin-angiotensin and kallikrein-kinin systems. Clin. Exp. Optom. 95: 282-289.

Schanstra JP, Neau E, Drogoz P, Arevalo Gomez MA, et al. (2002). In vivo bradykinin B2 receptor activation reduces renal fibrosis. J. Clin. Invest. 110: 371-379.

Tan Y, Hutchison FN and Jaffa AA (2004). Mechanisms of angiotensin II-induced expression of B2 kinin receptors. Am. J. Physiol. Heart Circ. Physiol. 286: H926-H932.

Tan Y, Wang B, Keum JS and Jaffa AA (2005). Mechanisms through which bradykinin promotes glomerular injury in diabetes. Am. J. Physiol. Renal Physiol. 288: F483-F492.

Tan Y, Keum JS, Wang B, McHenry MB, et al. (2007). Targeted deletion of B2-kinin receptors protects against the development of diabetic nephropathy. Am. J. Physiol. Renal Physiol. 293: F1026-F1035.

Tang SC, Leung JC and Lai KN (2011). The kallikrein-kinin system. Contrib. Nephrol. 170: 145-155.

Tomita H, Sanford RB, Smithies O and Kakoki M (2012). The kallikrein-kinin system in diabetic nephropathy. Kidney Int. 81: 733-744.

Wright JK, Botha JH and Naidoo S (2008). Influence of the kallikrein-kinin system on prostate and breast tumour angiogenesis. Tumour Biol. 29: 130-136.

Zhang JJ, Bledsoe G, Kato K, Chao L, et al. (2004). Tissue kallikrein attenuates salt-induced renal fibrosis by inhibition of oxidative stress. Kidney Int. 66: 722-732. 